\title{
On the Lyapunov exponents of the asteroidal motion subject to resonances and encounters
}

\author{
Ivan I. Shevchenko \\ Pulkovo Observatory of the Russian Academy of Sciences, Pulkovskoje ave. 65-1, \\ St. Petersburg 196140, Russia \\ email: iis@gao.spb.ru
}

\begin{abstract}
In theoretical as well as practical issues of the asteroidal hazard problem, it is important to be able to assess the degree of predictability of the orbital motion of asteroids. Some asteroids move in a virtually predictable way, others do not. The characteristic time of predictability of any motion is nothing but the Lyapunov time (the reciprocal of the maximum Lyapunov exponent) of the motion. In this report, a method of analytical estimation of the maximum Lyapunov exponents of the orbital motion of asteroids is described in application for two settings of the problem. Namely, the following two types of the motion are considered: (1) the motion close to the ordinary or three-body mean motion resonances with planets, and (2) the motion in highly eccentric orbits subject to moderately close encounters with planets. Whatever different these settings may look, the analytical treatment is universal: it is performed within a single framework of the general separatrix map theory. (Recall that the separatrix maps describe the motion near the separatrices of a nonlinear resonance.) The analytical estimates of the Lyapunov times are compared to known numerical ones, i.e., to known estimates obtained by means of numerical integration of the orbits.
\end{abstract}

Keywords. Chaos, Lyapounov exponents, resonances, close approaches

\section{Introduction}

Assessment of the degree of predictability of the orbital motion of asteroids and other potentially hazardous objects is one of the most complicated aspects of the asteroidal hazard problem. Some asteroids move in a virtually predictable way, others do not. Whipple (1995) wrote: "The existence of a significant population of extremely chaotic Earth-crossing asteroids must be factored into the thinking about the potential hazard posed by these objects. An asteroid with a Lyapunov time of 20 years may be considered as an example. If the initial error in its position is $100 \mathrm{~km}$ (a very optimistic assumption) then that error will grow to one Earth radius in 83 years and to an Earth-Moon distance in 165 years. Assessments of the threat from specific objects like this can be made for only short spans of time."

Generally, the estimation of the Lyapunov exponents is one of the most important tools in the study of chaotic motion (Lichtenberg \& Lieberman (1992)), in particular in celestial mechanics. The Lyapunov exponents characterize the mean rate of exponential divergence of trajectories close to each other in phase space; in the Hamiltonian systems, nonzero Lyapunov exponents indicate chaotic character of motion, while the maximum Lyapunov exponent equal to zero signifies regular (periodic or quasi-periodic) motion. The Lyapunov time (quantity reciprocal to the maximum Lyapunov exponent) gives the characteristic time of predictable dynamics.

The development of methods of numerical computation of the Lyapunov exponents has more than a thirty year history (see reviews in Froeschlé (1984), Lichtenberg \& Lieberman (1992)). On the contrary, methods of analytical estimation of the Lyapunov 
exponents started to be developed only recently (Holman \& Murray (1996), Murray \& Holman (1997), Shevchenko (2000a), Shevchenko (2002), Shevchenko (2004a)).

In this report, a method of analytical estimation of the maximum Lyapunov exponents of the orbital motion of asteroids is described in application for two settings of the problem. Namely, the following two types of the motion are considered: (1) the motion close to the ordinary or three-body mean motion resonances with planets, and (2) the motion in highly eccentric orbits subject to moderately close encounters with planets. Whatever different these settings may look, the analytical treatment is universal: it is performed within a single framework of the general separatrix map theory.

The analytical estimates of the Lyapunov times are compared to known numerical ones, i.e., to known estimates obtained by means of numerical integration of the orbits.

\section{The model of perturbed resonance}

Under general conditions (Chirikov (1977), Chirikov (1979), Lichtenberg \& Lieberman (1992)), a model of nonlinear resonance is provided by the Hamiltonian of the nonlinear pendulum with periodic perturbations:

$$
H=\frac{\mathcal{G} p^{2}}{2}-\mathcal{F} \cos \varphi+a \cos (\varphi-\tau)+b \cos (\varphi+\tau) .
$$

The first two terms in Eq. (2.1) represent the Hamiltonian $H_{0}$ of the unperturbed pendulum; $\varphi$ is the pendulum angle (the resonance phase angle), $p$ is the momentum. The periodic perturbations are given by the last two terms; $\tau$ is the phase angle of perturbation: $\tau=\Omega t+\tau_{0}$, where $\Omega$ is the perturbation frequency, and $\tau_{0}$ is the initial phase of the perturbation. The quantities $\mathcal{F}, \mathcal{G}, a, b$ are constants. The frequency of the pendulum small-amplitude oscillations $\omega_{0} \equiv(\mathcal{F} \mathcal{G})^{1 / 2}$.

An example of section of phase space of the Hamiltonian $(2.1)$ at $\tau=0 \bmod 2 \pi$ is shown in Fig. $1\left(\Omega=5, \omega_{0}=1, a=b, \varepsilon \equiv \frac{a}{\mathcal{F}}=0.5\right)$. This is a chaotic resonance triplet.

The motion near the separatrices of Hamiltonian (2.1) is described by the so-called separatrix algorithmic map (Shevchenko (1999)):

$$
\begin{aligned}
& \text { if } w_{n}<0 \text { and } W=W^{-} \text {then } W=W^{+}, \\
& \text {if } w_{n}<0 \text { and } W=W^{+} \text {then } W=W^{-} ; \\
& w_{n+1}=w_{n}-W \sin \tau_{n}, \\
& \tau_{n+1}=\tau_{n}+\lambda \ln \frac{32}{\left|w_{n+1}\right|} \quad(\bmod 2 \pi) ;
\end{aligned}
$$

with the parameters

$$
\begin{gathered}
\lambda=\frac{\Omega}{\omega_{0}}, \\
W^{+}(\lambda, \eta)=\varepsilon \lambda\left(A_{2}(\lambda)+\eta A_{2}(-\lambda)\right), \\
W^{-}(\lambda, \eta)=\varepsilon \lambda\left(\eta A_{2}(\lambda)+A_{2}(-\lambda)\right),
\end{gathered}
$$

$\varepsilon=\frac{a}{\mathcal{F}}, \eta=\frac{b}{a}$. The Melnikov-Arnold integral $A_{2}(\lambda)$ is given by the relation

$$
A_{2}(\lambda)=4 \pi \lambda \frac{\exp (\pi \lambda / 2)}{\sinh (\pi \lambda)},
$$

see (Chirikov (1979), Shevchenko (1998b), Shevchenko (2000b)).

The quantity $w$ denotes the relative (with respect to the separatrix value) pendulum energy: $w \equiv \frac{H_{0}}{\mathcal{F}}-1$. The variable $\tau$ retains its meaning of the phase angle of perturbation. 


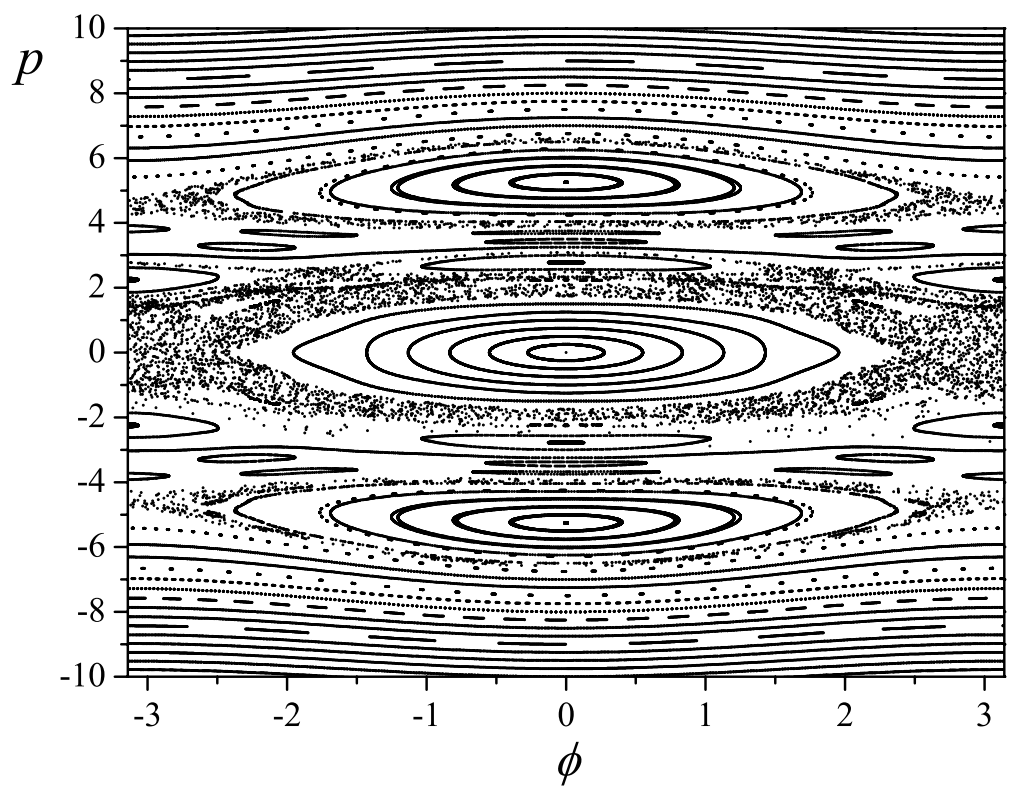

Figure 1. An example of a chaotic resonance triplet.

One iteration of map (2.2) corresponds to one half-period of pendulum's libration or one period of its rotation.

If $a=b$ (symmetric case), the separatrix algorithmic map reduces to the well-known ordinary separatrix map

$$
\begin{aligned}
& w_{i+1}=w_{i}-W \sin \tau_{i}, \\
& \tau_{i+1}=\tau_{i}+\lambda \ln \frac{32}{\left|w_{i+1}\right|} \quad(\bmod 2 \pi),
\end{aligned}
$$

written in the present form in (Chirikov (1977), Chirikov (1979)); the expression for $W$ (Shevchenko (1998b), Shevchenko (2000b)) is

$$
W=\varepsilon \lambda\left(A_{2}(\lambda)+A_{2}(-\lambda)\right)=4 \pi \varepsilon \frac{\lambda^{2}}{\sinh \frac{\pi \lambda}{2}} .
$$

Formula (2.7) differs from that given in (Chirikov (1979), Lichtenberg \& Lieberman (1992)) by the term $A_{2}(-\lambda)$, which is small for $\lambda \gg 1$. However, its contribution is significant for $\lambda$ small (Shevchenko (1998b)), i.e., in the case of adiabatic chaos.

An equivalent form of Eqs. (2.6), used, e.g., in (Chirikov \& Shepelyansky (1984), Shevchenko (1998a)), is

$$
\begin{aligned}
& y_{i+1}=y_{i}+\sin x_{i}, \\
& x_{i+1}=x_{i}-\lambda \ln \left|y_{i+1}\right|+c \quad(\bmod 2 \pi),
\end{aligned}
$$

where $y=w / W, x=\tau+\pi ;$ and

$$
c=\lambda \ln \frac{32}{|W|} .
$$

The applicability of the theory of separatrix maps for description of the motion near the separatrices of the perturbed nonlinear resonance in the full range of the relative frequency of perturbation, including its low values, was discussed and shown to be legitimate in (Shevchenko (2000b)). 
The half-width $y_{b}$ of the main chaotic layer of the separatrix map (2.8) in the case of the least perturbed border of the layer is presented as a function of $\lambda$ in Fig. 1 in (Shevchenko (2004a)). The observed dependence follows the piecewise linear law with a transition point at $\lambda \approx 1 / 2$. This transition takes place not only in what concerns the width of the layer, but also in other characteristics of the motion, in particular, in the maximum Lyapunov exponent. The clear sharp transition at this point manifests a qualitative distinction between two types of dynamics, "slow" and "fast" chaos.

\section{The method of analytical estimation of Lyapunov times}

In (Shevchenko (2000a), Shevchenko (2002)), a method for estimation of the maximum Lyapunov exponent of the chaotic motion in the vicinity of separatrices of perturbed nonlinear resonance was derived in the framework of the separatrix map theory. Following the general approach (Shevchenko (2000a), Shevchenko (2002)), we represent the maximum Lyapunov exponent $L$ of the motion in the main chaotic layer of system (2.1) as the ratio of the maximum Lyapunov exponent $L_{s x}$ of its separatrix map and the average period $T$ of rotation (or, equivalently, the average half-period of libration) of the resonance phase $\varphi$ inside the layer. For convenience, we introduce a non-dimensional quantity $T_{s x}=\Omega T$. Then the general expression for $L$ is

$$
L=\Omega \frac{L_{s x}}{T_{s x}} .
$$

The quantity $T_{L} \equiv L^{-1}$, by definition, is the Lyapunov time.

We consider four generic resonance types: the fastly chaotic resonance triplet, fastly chaotic resonance doublet, slowly chaotic resonance triplet, slowly chaotic resonance doublet (we call them, respectively, the "ft", "fd", "st", "sd" resonance types).

\subsection{Fast chaos. Resonance triplet}

Consider the case of $a=b, \lambda>1 / 2$. This means that there is a symmetric triad of interacting resonances (first condition), and chaos is fast (second condition).

The case of the fastly chaotic triad is completely within the range of applicability of the method presented in (Shevchenko (2000a), Shevchenko (2002)). The perturbed nonlinear resonance is modelled by Hamiltonian (2.1) with $a=b$. Following (Shevchenko (2000a), Shevchenko (2002)), we take the dependence of the maximum Lyapunov exponent of the separatrix map (2.8) upon $\lambda$ in the form

$$
L_{s x}(\lambda) \approx C_{h} \frac{2 \lambda}{1+2 \lambda}
$$

where $C_{h} \approx 0.80$ is a constant (Shevchenko (2004b)).

The average increment of $\tau$ (proportional to the average rotation period, or libration half-period) inside the chaotic layer is (Chirikov (1979), Shevchenko (2000a), Shevchenko (2002)):

$$
T_{s x}(\lambda, W) \approx \lambda \ln \frac{32 e}{\lambda|W|}
$$

where $e$ is the base of natural logarithms. From Eq. (3.1), one has for the Lyapunov time for the "ft" resonance type:

$$
T_{L}=\frac{T_{p e r t}}{2 \pi} \frac{T_{s x}}{L_{s x}} \approx T_{p e r t} \frac{(1+2 \lambda)}{4 \pi C_{h}} \ln \frac{32 e}{\lambda|W|},
$$

where $T_{\text {pert }}=2 \pi / \Omega$ is the period of perturbation. 


\subsection{Fast chaos. Resonance doublet}

The previous analysis of the symmetric case $a=b$ sets a foundation for an analysis of the more general asymmetric case $a \neq b$, since the Lyapunov exponents in the asymmetric case can be found by averaging the contributions of the separate components of the chaotic layer (Shevchenko (2004a)).

Calculation of the average constitutes a complicated problem. In particular, one should know the relative average times of residence of the system in three different components of the layer corresponding to direct rotation, reverse rotation, and libration of the pendulum. The relative times of residence depend on the asymmetry of perturbation. A simple heuristic method of averaging was proposed in (Shevchenko (2000a), Shevchenko (2002)), but rigorous solution is still far from being found.

In view of these difficulties, we consider the limit case of $a$ or $b$ equal to zero. It means that one of the two perturbing resonances simply does not exist, and instead of the resonance triad we have a duad.

If $\lambda>1 / 2$, the equality $b=0$ implies $\left|W^{-}\right| \ll\left|W^{+}\right|$, and, vice versa, $a=0$ implies $\left|W^{-}\right| \gg\left|W^{+}\right|$. We designate the dominating quantity by $W$.

Consider first the libration side of the chaotic layer. Then $W^{-}$and $W^{+}$alternate (replace each other) at each iteration of the separatrix algorithmic map (2.2). It is straightforward to show that, if $W^{-}$or $W^{+}$is equal to zero, the separatrix algorithmic map (2.2) on the doubled iteration step reduces to the ordinary separatrix map (2.6) with the doubled value of $\lambda$ and the same non-zero value of $W$. One iteration of the new map corresponds to two iterations of the old one. Since the half-width of the chaotic layer of map (2.6) is $\approx \lambda W$ (Chirikov (1979), Shevchenko (2004a)), the layer's extent in $w$ on the side of librations doubles, it becomes $\approx 2 \lambda W$. Note that the parameters $\lambda$ and $W$ are considered here as independent from each other.

Consider then the circulation sides of the chaotic layer. The side corresponding to reverse (or direct) rotations does not exist, if $W^{-}$(or, respectively, $W^{+}$) is equal to zero; its measure is zero. The other side, corresponding to direct (or reverse) rotations is described by the ordinary separatrix map (2.6) with the parameters $\lambda, W$; its extent in $w$ is $\approx \lambda W$.

The averaged (over the whole layer) value of the maximum Lyapunov exponent is the sum of weighted contributions of the layer components corresponding to the librations, direct rotations and reverse rotations of the pendulum. The weights are directly proportional to the times that the trajectory spends in the components, and, via supposed approximate ergodicity, to the relative measures of the components in phase space. Taking into account the just made estimates of the widths of the chaotic layer's components in the duad case, one can expect that the relative weights of librations and circulations in the "fd" case are respectively 4 and 1.

Hence the formula for the Lyapunov exponent for the "fd" resonance type is

$$
L=\frac{\Omega}{\mu_{l i b r}+1}\left(\mu_{l i b r} \frac{L_{s x}(2 \lambda)}{T_{s x}(2 \lambda, W)}+\frac{L_{s x}(\lambda)}{T_{s x}(\lambda, W)}\right),
$$

and

$$
T_{L}=\frac{T_{p e r t}}{2 \pi} \cdot \frac{\mu_{l i b r}+1}{\mu_{l i b r} \frac{L_{s x}(2 \lambda)}{T_{s x}(2 \lambda, W)}+\frac{L_{s x}(\lambda)}{T_{s x}(\lambda, W)}},
$$

where $\mu_{l i b r} \approx 4$, and $W, L_{s x}, T_{s x}$ are given by formulas $(2.7,3.2,3.3)$. 


\subsection{Slow chaos. Resonance triplet}

In the case of $\lambda<1 / 2$, the diffusion across the layer is slow, and on a short time interval the phase point of the ordinary separatrix map (2.8) follows close to some current curve. We call this curve guiding. Let us derive an analytical expression for the guiding curve with an irrational winding number far enough from the main rationals. We approximate the winding number by the rationals $m / n$. Thus $c \approx 2 \pi m / n$. Noticing that at an iteration $n$ of the map the phase point hits in a small neighborhood of the starting point, one obtains for the derivative:

$$
\begin{aligned}
\frac{d y}{d x} & =\frac{1}{n c-2 \pi m} \sum_{k=0}^{n-1} \sin (x+k c)= \\
& =\frac{1}{n c-2 \pi m} \sin \frac{n c}{2} \operatorname{cosec} \frac{c}{2} \sin \left(x+\frac{n-1}{2} c\right) .
\end{aligned}
$$

Integrating and passing to the limit $n \rightarrow \infty$, one obtains:

$$
y=-\frac{1}{2} \operatorname{cosec} \frac{c}{2} \cos \left(x-\frac{c}{2}\right)+\mathcal{C},
$$

where $\mathcal{C}$ is an arbitrary constant of integration.

The motion is chaotic only when the curve (3.8) crosses the singular line $y=0$. Hence the half-width of the chaotic layer is $y_{b}=\left|\operatorname{cosec} \frac{c}{2}\right|$. Averaging (by taking an integral analytically) the quantity $-\ln \left|y_{i+1}\right|$ (equal to $(\langle\Delta x\rangle-c) / \lambda$, Eqs. (2.8)), where $y_{i+1}$ is substituted by $y$ of Eq. (3.8), over the chaotic layer in the derived boundaries, we find the approximate analytical expression for $\Theta \equiv\left(T_{s x}-c\right) / \lambda$ :

$$
\Theta \approx \ln \left|4 \sin \frac{c}{2}\right|
$$

Then, we need an expression for $L_{s x}(\lambda)$. We explore the $\lambda$ dependence of $L_{s x}$ in a numerical experiment. At each step in $\lambda$ (namely, $\Delta \lambda=0.005, \lambda \geqslant 0.005$ ) we find the value of $c$ corresponding to the case of the least perturbed layer and plot the value of $L_{s x}$. At $\lambda<0.3$, the dependence turns out to be practically linear. The linear fit $L_{s x}(\lambda)=a \lambda$ gives $a=1.01132 \pm 0.00135$, and the correlation coefficient $R=0.9998$.

We set $L_{s x} \approx \lambda$ for the generic (non-resonant) values of $c$ and for $\lambda<1 / 2$. Then, from Eqs. $(3.1,3.9)$ one has the following approximate formula for the maximum Lyapunov exponent:

where $c=\lambda \ln \frac{32}{|W|}$ (Eq. (2.9)).

$$
L \approx \frac{\Omega}{\ln \left|4 \sin \frac{c}{2}\right|+\frac{c}{\lambda}}
$$

For $\lambda \ll 1$ one has $W \approx 8 \varepsilon \lambda$, hence the formula for the Lyapunov time for the "st" resonance type:

$$
T_{L} \approx \frac{T_{\text {pert }}}{2 \pi} \ln \left|\frac{16}{\varepsilon \lambda} \sin \left(\frac{\lambda}{2} \ln \frac{4}{|\varepsilon| \lambda}\right)\right| .
$$

\subsection{Slow chaos. Resonance doublet}

Utilizing the approximation of the Melnikov-Arnold integral $A_{2}(\lambda) \approx 2 \pi \lambda+4$ at $\lambda \ll$ $1, \eta=0$, one has: $W^{ \pm} \approx \varepsilon \lambda(4 \pm 2 \pi \lambda) \approx 4 \varepsilon \lambda$. So, in the "sd" case, the separatrix 


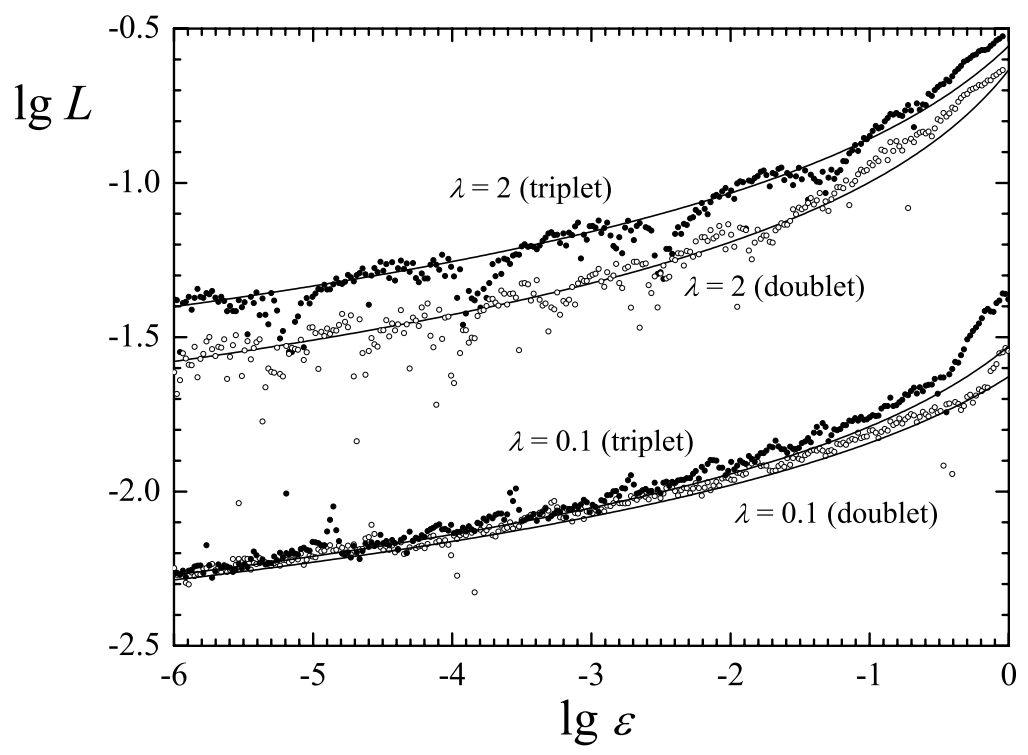

Figure 2. The maximum Lyapunov exponent of the chaotic motion of system (2.1) in dependence on the magnitude of perturbation: the results of direct computation (circles) and the theoretical curves.

algorithmic map (2.2) degenerates to the ordinary separatrix map (2.6) with $W \approx 4 \varepsilon \lambda$, i.e., mathematically the case is equivalent to the "st" case, but with a different (halved) value of $W$.

Following the lines of the previous Subsection, it is then straightforward to write down the formula for the Lyapunov time for the "sd" resonance type:

$$
T_{L} \approx \frac{T_{\text {pert }}}{2 \pi} \ln \left|\frac{32}{\varepsilon \lambda} \sin \left(\frac{\lambda}{2} \ln \frac{8}{|\varepsilon| \lambda}\right)\right| .
$$

\subsection{Theory versus numerical experiment}

To check the theory, the Lyapunov exponents of the chaotic motion near the separatrices of Hamiltonian (2.1) have been directly computed by means of the program package (Shevchenko \& Kouprianov (2002), Kouprianov \& Shevchenko (2003)) utilizing the HQRB method by von Bremen, Udwadia \& Proskurowski (1997). The power of this method is far greater than that necessary in the present computation. It allows one to evaluate the full Lyapunov spectrum of a multidimensional system. The spectrum of our system consists of a sole pair of Lyapunov exponents - the maximum one and its negative counterpart. The integration of the equations of motion has been performed by the integrator by Hairer, Nørsett \& Wanner (1987). It is an explicit 8th order Runge-Kutta method due to Dormand and Prince, with the step size control. We choose the time unit in such a way that $\omega_{0}=1$.

The results of the computations for $\lambda=0.1$ and 2 are shown in Fig. 2 (circles). The integration time interval has been chosen to be equal to $10^{6}$. This is sufficient for the computed values of the Lyapunov exponents to saturate in each case. Local wave-like patterns represent prominent features of the constructed dependences; they are conditioned by the process of encountering resonances, while $\varepsilon$ changes. 
The theoretical dependences are shown in Fig. 2 as solid curves. $T_{L}$ are given by formulas $(3.4,3.6,3.11,3.12) ; L=1 / T_{L}$. Close correspondence is observed between the theory and experimental data for each value of $\lambda$.

\section{Lyapunov times of the asteroidal motion subject to resonances}

The mean motion resonances and secular resonances represent the main classes of the orbital resonances in the motion of asteroids. The mean motion ones represent commensurabilities of the periods of the motion of asteroids and planets. There are two main subclasses of the mean motion resonances: ordinary (two-body) resonances and threebody resonances.

\subsection{Ordinary mean motion resonances}

The Hamiltonian of the motion of a zero-mass test particle in the gravitational field of the Sun and Jupiter, in the plane of Jupiter's orbit, in the vicinity of a mean motion resonance with Jupiter can be represented in some approximation in the form (Holman \& Murray (1996), Murray \& Holman (1997)):

$$
H=\frac{1}{2} \beta \Lambda^{2}-\sum_{p=0}^{q} \phi_{k+q, k+p, k} \cos (\psi-p \omega)
$$

where $\beta=3 k^{2} / a^{2}, \Lambda=\Psi-\Psi_{\text {res }}, \Psi=\left(\mu_{1} a\right)^{1 / 2} / k, \Psi_{\text {res }}=\left(\mu_{1}^{2} /\left(k^{2}(k+q) n_{J}\right)\right)^{1 / 3}, \mu_{1}=$ $1-\mu, \omega \equiv-\varpi$ (i.e., $\omega$ is minus the longitude of asteroid's perihelion; its time derivative is assumed to be constant); $a$ and $e$ are asteroid's semimajor axis and eccentricity. The integer non-negative numbers $k$ and $q$ define the resonance: the ratio $(k+q) / k$ equals the ratio of mean motions of an asteroid and Jupiter in the exact resonance. The phase $\psi \equiv k l-(k+q) l_{J}$, where $l$ and $l_{J}$ are the mean longitudes of an asteroid and Jupiter.

Here the units are chosen in such a way that the total mass (Sun plus Jupiter), the gravitational constant, Jupiter's semimajor axis $a_{J}$ are all equal to one; $\mu=1 / 1047.355$, $\mu_{1}=1-\mu$. Jupiter's mean longitude $l_{J}=n_{J} t$, eccentricity $e_{J}=0.048$. Jupiter's mean motion $n_{J}=1$, i.e., the time unit equals $\frac{1}{2 \pi}$ th part of Jupiter's orbital period.

According to Eq. (4.1), the resonance $(k+q) / k$ splits in a cluster of $q+1$ subresonances $p=0,1, \ldots, q$. The coefficients of the resonant terms are

$$
\left|\phi_{k+q, k+p, k}\right| \approx \frac{\mu}{q \pi a_{J}}\left(\begin{array}{c}
q \\
p
\end{array}\right)\left(\frac{\epsilon}{2}\right)^{p}\left(\frac{\epsilon_{J}}{2}\right)^{q-p},
$$

where $\epsilon=e a_{J} /\left|a-a_{J}\right|, \epsilon_{J}=e_{J} a_{J} /\left|a-a_{J}\right|$ (Holman \& Murray (1996), Murray \& Holman (1997)). The approximation (4.2) is good, if $\epsilon q<1$ (Holman \& Murray (1996)). Besides, the model is restricted to the resonances of relatively high order, $q \geqslant 2$.

The signs of the coefficients $\phi_{k+q, k+p, k}$ alternate with changing $p$, so, the coefficients with numbers $p$ and $p+2$ are always of the same sign. This means that whatever is the choice of the guiding resonance in the multiplet, its closest neighbors have coefficients of equal signs, and $\eta$ is always non-negative.

The frequency of small-amplitude oscillations at the subresonance $p$

$$
\omega_{0}=\left(\beta\left|\phi_{k+q, k+p, k}\right|\right)^{1 / 2} \approx \frac{a_{J}}{\left|a-a_{J}\right|} n_{J}\left(\mu_{1} \mu \frac{4 q}{3 \pi}\left(\begin{array}{c}
q \\
p
\end{array}\right)\left(\frac{a}{a_{J}}\right)\left(\frac{\epsilon}{2}\right)^{p}\left(\frac{\epsilon_{J}}{2}\right)^{q-p}\right)^{1 / 2}
$$


Table 1. Numerical and analytical estimates of Lyapunov times for ordinary mean motion resonances, $T_{L}$ in years, $e=0.1$

\begin{tabular}{rcccc}
\hline$\frac{k+q}{k}$ & $\lambda$ & $\lg T_{L}^{\text {num }} \dagger$ & $\lg T_{L}^{\text {theor }}$ & Res. type \\
\hline $3 / 1$ & 0.093 & $3.8-4.3$ & 4.3 & $\mathrm{sd}$ \\
$5 / 2$ & 0.192 & $3.5-3.8$ & 4.1 & $\mathrm{st}$ \\
$7 / 3$ & 0.415 & $3.8-4.2$ & 4.0 & $\mathrm{st}$ \\
$9 / 4$ & 0.932 & $3.9-4.3$ & 4.2 & $\mathrm{fd}^{*}$ \\
$11 / 5$ & 1.970 & $3.9-4.3$ & 4.3 & $\mathrm{ft}^{*}$ \\
$9 / 5$ & 0.323 & $3.6-3.8$ & 3.7 & $\mathrm{st}^{*}$ \\
$7 / 4$ & 0.166 & $3.2-3.3$ & 3.7 & $\mathrm{st}^{*}$ \\
$12 / 7$ & 0.594 & $3.6-4.0$ & 3.9 & $\mathrm{fd}^{*}$ \\
$5 / 3$ & 0.101 & $2.5-3.3$ & 3.7 & $\mathrm{sd}^{*}$ \\
$8 / 5$ & 0.156 & $2.5-3.3$ & 3.6 & $\mathrm{st}^{*}$ \\
$11 / 7$ & 0.264 & $3.3-3.6$ & 3.5 & $\mathrm{st}^{*}$ \\
\hline
\end{tabular}

$\dagger$ Morbidelli \& Nesvorný (1999), Holman \& Murray (1996)

and the perturbation frequency

$$
\Omega=\dot{\omega} \approx \frac{\mu_{1} \mu}{2 \pi} n_{J}\left(\frac{a}{a_{J}}\right)^{1 / 2}\left(\frac{a_{J}}{a-a_{J}}\right)^{2},
$$

cf. (Holman \& Murray (1996), Murray \& Holman (1997)). The ratio of $\Omega$ and $\omega_{0}$ gives the value of $\lambda$.

Now we are able to apply the theory developed in Section 3. For comparison, we take the data on the numerical (based on integrations) values of $T_{L}$ for the motion near mean motion resonances from Fig. 1 in (Morbidelli \& Nesvorný (1999)) and Fig. 6 in (Holman \& Murray (1996)). The theoretical estimates are made by means of formulas $(3.4,3.6$, $3.11,3.12$ ). Before they are used, the guiding resonance in the multiplet is identified (it has the maximum value of $\left|\phi_{k+q, k+p, k}\right|$ ), and its two closest neighbors are considered as the perturbing resonances. Then, the formula is chosen in accord with the resonance type (fastly chaotic triad "ft", fastly chaotic duad "fd", slowly chaotic triad "st", or slowly chaotic duad "sd"). If the amplitudes of the neighbors differ from each other less than twice, the model resonance is considered to be a triad, otherwise a duad. Those resonances which have $\epsilon q>1$ are marked in Table 1 by an asterisk.

The analytical maximum Lyapunov exponent estimates are generally in agreement with the numerical ones. However, some differences can be clearly seen, especially in the domain of slow chaos. This should be attributed to the imperfectness of model (4.1), and mainly to the fact that the coefficients $\phi_{k+q, k+p, k}$ are treated as constants. They fix the frequencies $\omega_{0}$ of small amplitude oscillations at subresonances, and when the period of perturbation is large in relation to the period of these oscillations, the variations of $\omega_{0}$ can have greater dynamical influence.

Also another effect can be of importance. The differential distribution, built by Shevchenko, Kouprianov \& Melnikov (2003) for a representative plane of starting values 
(the trajectories were computed by the Wisdom map (Wisdom (1983)) in the planar elliptic restricted three-body problem), demonstrates that the maximum Lyapunov exponent of the trajectories near the $3 / 1$ mean-motion resonance with Jupiter has two, and not one, preferable numerical values: the distribution has a bimodal peak structure. This signifies that there are two distinct domains of chaos in phase space; thus the perturbed pendulum model as applied to this low-order mean motion resonance turns out to be too approximate. This example shows also that generally a closer look at the numerical data may be necessary when comparing it with theory.

Now consider an example of an estimate for a real asteroid, namely (522) Helga. This object is famous to be the first example of "stable chaos" among asteroids (Milani \& Nobili (1993)): while its Lyapunov time is relatively small (6900 years), its orbit does not exhibit any gross changes on cosmogonic time scales, according to numerical experiments. It is known to be in the $12 / 7$ mean motion resonance.

Let us apply our method. The necessary data on $a, e$, the perihelion frequency $g=\dot{\varpi}$ are taken from the "numb.syn" catalogue (Knežević \& Milani (2000)) of the AstDyS web service†. $T_{\text {pert }}$ is defined by the value of $g$. We find that the guiding subresonance in the resonance sextet is the third one $(p=2)$, consequently the perturbing neighbors in our model have the numbers $p=1$ and 3 . The quantity $\epsilon q=0.624<1$, so there are no problems with the potential model. The derived separatrix map parameters are: $\lambda=2.325, \eta=0.812$, consequently the model resonance type is the fastly chaotic triplet "ft". Applying formula (3.4), one has $T_{L}=9700$ years. The agreement with the values, obtained in integrations in the full problem, (6900 years (Milani \& Nobili (1993)), 6860 years (AstDyS)) should be considered as satisfactory.

\subsection{Three-body mean motion resonances}

An important role in the orbital dynamics of bodies of the Solar system, in particular asteroids, is played by the so-called three-body resonances (Murray, Holman \& Potter (1998), Nesvorný \& Morbidelli (1998), Nesvorný \& Morbidelli (1999)). In the case of a three-body resonance, the resonant phase is a combination of angular elements of the orbits of three bodies (a test one and two perturbing ones; e.g., an asteroid, Jupiter, and Saturn).

The three-body resonances can be described by the perturbed pendulum model (Murray, Holman \& Potter (1998), Nesvorný \& Morbidelli (1998), Nesvorný \& Morbidelli (1999)). The Hamiltonian of the motion of a zero-mass test particle near a three-body resonance $\left\{m_{J} m_{S} m\right\}$ with Jupiter and Saturn in the planar-elliptic problem can be expressed, in some approximation, in the following form (Nesvorný \& Morbidelli (1999)):

$$
H=\alpha S^{2}+\sum_{p_{J}, p_{S}, p} \beta_{p_{J} p_{S} p} \cos \sigma_{p_{J} p_{S} p}
$$

where the conjugated to $S$ resonance argument $\sigma_{p_{J} p_{S} p}=m_{J} l_{J}+m_{S} l_{S}+m l+p_{J} \varpi_{J}+$ $p_{S} \varpi_{S}+p \varpi$ (it is assumed that the time derivatives of $l_{J}, l_{S}, \varpi_{J}, \varpi_{S}$ are constants), $\alpha=-(3 / 2) n^{2} a_{r e s}^{-2}$. Analytical expressions $\beta_{p_{J} p_{S} p}(e)$ for some important three-body resonances are given in Tables 3-6 in (Nesvorný \& Morbidelli (1999)). It is clear from Eq. (4.5) that the three-body resonance $\left\{m_{J} m_{S} m\right\}$ splits in a cluster of subresonances with various $\left\{p_{J} p_{S} p\right\}$ combinations.

$\dagger$ http://hamilton.dm.unipi.it/cgi-bin/astdys/ 
Table 2. Numerical and analytical estimates of Lyapunov times for asteroids in three-body mean motion resonances

\begin{tabular}{lccccccc}
\hline Asteroid & $\begin{array}{c}\text { Resonance } \\
\left\{m_{J} m_{S} m\right\}\end{array}$ & $\lambda$ & $\begin{array}{c}T_{L}^{\text {num }} \\
\mathrm{yr} \dagger\end{array}$ & $\begin{array}{c}T_{L}^{\text {num }} \\
\mathrm{yr} \ddagger\end{array}$ & $\begin{array}{c}T_{L}^{\text {theor }} \\
\mathrm{yr}\end{array}$ & $\begin{array}{c}\text { Res. } \\
\text { type }\end{array}$ \\
\hline 258 Tyche & $2+2-1$ & 0.536 & 35900 & - & 43100 & $\mathrm{ft}$ \\
485 Genua & $3-1-1$ & 0.376 & 6550 & 6500 & 35700 & $\mathrm{sd}$ \\
1642 Hill & $3-1-1$ & 0.643 & 36100 & - & 43300 & $\mathrm{fd}$ \\
936 Kunigunde & $6+1-3$ & 0.624 & 22200 & - & 54600 & $\mathrm{fd}$ \\
490 Veritas & $5-2-2$ & 0.546 & 10200 & 8500 & 9100 & $\mathrm{fd}$ \\
2039 Paine-Gaposchkin & $5-2-2$ & 0.449 & 22000 & - & 6020 & $\mathrm{sd}$ \\
3460 Ashkova & $5-2-2$ & 0.433 & 65100 & 8300 & 5940 & $\mathrm{sd}$ \\
\hline
\end{tabular}

$\dagger$ AstDyS

† Nesvorný \& Morbidelli (1998), Nesvorný \& Morbidelli (1999), Milani, Nobili \& Knežević (1997)

The frequency of small-amplitude oscillations at the subresonance $\left\{p_{J} p_{S} p\right\}$ is (Nesvorný \& Morbidelli (1999)):

$$
\omega_{0}=2 \pi n\left(3 \beta_{p_{J} p_{S} p}\right)^{1 / 2} a_{r e s}^{-1} .
$$

The perturbation frequency $\Omega$ is generally an algebraic combination of perihelion frequencies of Jupiter, Saturn and the asteroid. The formula for this combination is defined by the choice of the guiding subresonance (see below). The ratio of $\Omega$ and $\omega_{0}$ gives the value of $\lambda$.

We consider the asteroids residing close to the three-body resonances studied in (Nesvorný \& Morbidelli (1999)), and utilize the analytical data in Tables 3-6 in (Nesvorný \& Morbidelli (1999)) on the coefficients of resonant terms. The theoretical estimates of Lyapunov times are made by means of formulas $(3.4,3.6,3.11,3.12)$. Before they are used, the guiding resonance in the multiplet is identified (it has the maximum value of $\left.\left|\beta_{p_{J} p_{S} p}\right|\right)$, and its two closest neighbors are considered as the perturbing resonances. Then, the formula is chosen in accord with the resonance type ("ft", "fd", "st", "sd").

On identification of the guiding resonances in the multiplets, it turns out that the three-body resonances under study subdivide in two distinct classes: those for which the perturbation frequency $\Omega$ in model $(2.1)$ is equal to $\dot{\varpi}-\dot{\varpi}_{J}$, and those for which it is equal to $\dot{\varpi}_{S}-\dot{\varpi}_{J}$. The resonances $5-2-2$ and $3-1-1$ belong to the first class, while $2+2-1$ and $6+1-3$ to the second. We use $\dot{\varpi}_{J}=4.257^{\prime \prime} / \mathrm{yr}$ and $\dot{\varpi}_{S}=28.243^{\prime \prime} / \mathrm{yr}$ (Bretagnon (1990)). The data on $\dot{\varpi}, a$, and $e$ are taken from the "numb.syn" catalogue (Knežević \& Milani (2000)) of the AstDyS web service.

The theoretical estimates, obtained in this way, are presented in Table 2. Some of them are in accord with the numerical ones (in particular, in the case of (490) Veritas), others are not. From the fact of disagreement in the cases of (485) Genua and (2039) Paine-Gaposchkin, one can judge that these objects do not, most probably, reside in the chaotic layers of the prescribed resonance multiplets. Thus the analytical estimation of Lyapunov times represents a promising tool for discerning between possible models of chaos in the motion of real asteroids, and, generally, celestial bodies. 


\section{Lyapunov times of the asteroidal motion subject to encounters}

\subsection{Lyapunov exponents of the motion described by the Kepler map}

Consider a map similar to Eqs. (2.8), but with a power-law phase increment instead of the logarithmic one:

$$
\begin{aligned}
& y_{i+1}=y_{i}+\sin x_{i}, \\
& x_{i+1}=x_{i}-\lambda\left|y_{i+1}\right|^{-\gamma}+c \quad(\bmod 2 \pi) .
\end{aligned}
$$

A number of mechanical and physical models are described by such maps. The case of $\gamma=3 / 2, c=0$ corresponds to the Kepler map. It was derived and analyzed in (Chirikov \& Vecheslavov (1986), Vecheslavov \& Chirikov (1988), Chirikov \& Vecheslavov (1989), Petrosky (1986)) in order to describe the chaotic motion of the Halley comet and, generally, the motion of comets in nearly parabolic orbits. The motion model consists in the assumption that the main perturbing effect of Jupiter is concentrated when the comet is close to the perihelion of its orbit. This effect is defined by the phase of encounter with Jupiter. The variable $y$ has the meaning of the normalized full energy of the comet, while $x$ is the normalized time. One iteration of the map corresponds to one orbital revolution of the comet.

Chirikov's constant $C_{h}^{g e n}$ for general separatrix map (5.1) with an arbitrary value of $\gamma$ is introduced in the same way as one for basic map (2.8): it is the least upper bound for the maximum Lyapunov exponent of the motion in the main chaotic layer of the map. The proper limit can be shown to exist in the same way as it was done in (Shevchenko (2004b)) for the ordinary separatrix map.

By means of linearization of map (5.1) in $y$ it is straightforward to see that the value of $y$ corresponding to the critical value of the stochasticity parameter $K=K_{G}$ of the approximating standard map is $y_{b}=\left(\gamma \lambda / K_{G}\right)^{\frac{1}{\gamma+1}}$, while the value of $y$ corresponding to $K=4$ is $y_{p}=(\gamma \lambda / 4)^{\frac{1}{\gamma+1}}$. The first of these values marks the border of the chaotic layer, while the second one roughly separates mostly "non-porous" and mostly "porous" parts of the layer. The ratio $y_{b} / y_{p} \approx 4^{\frac{1}{\gamma+1}}$; hence the contribution of the porous part to the value of the maximum Lyapunov exponent in the layer becomes negligible with $\gamma$ increasing. This makes the estimation of Chirikov's constant in the case of general separatrix map (5.1) with $\gamma>0$ more precise than in the case of map (2.8), because the contribution of the porous part, which is small here, is most uncertain.

An expression for Chirikov's constant $C_{h}^{g e n}$ for the general separatrix map is derived from Eq. (9) in (Shevchenko (2004b)) in the same way as in the case of Chirikov's constant $C_{h}$ for the basic separatrix map (cf. Shevchenko (2004b)), except the change of the variable and the expression for layer's half-width are different. The resulting expression is

$$
C_{h}^{g e n}(\gamma)=\frac{K_{G}^{\frac{1}{\gamma+1}}}{(\gamma+1) \sigma(\gamma)} \int_{K_{G}}^{\infty} L(K) \mu(K) \frac{d K}{K^{\frac{\gamma+2}{\gamma+1}}}
$$

where

$$
\sigma(\gamma)=\frac{K_{G}^{\frac{1}{\gamma+1}}}{\gamma+1} \int_{K_{G}}^{\infty} \mu(K) \frac{d K}{K^{\frac{\gamma+2}{\gamma+1}}}
$$

The functions $L(K)$ and $\mu(K)$ (the maximum Lyapunov exponent of the standard map and the measure of the chaotic component in phase space of the standard map, both in dependence on the stochasticity parameter) were computed in (Shevchenko (2004b)). 


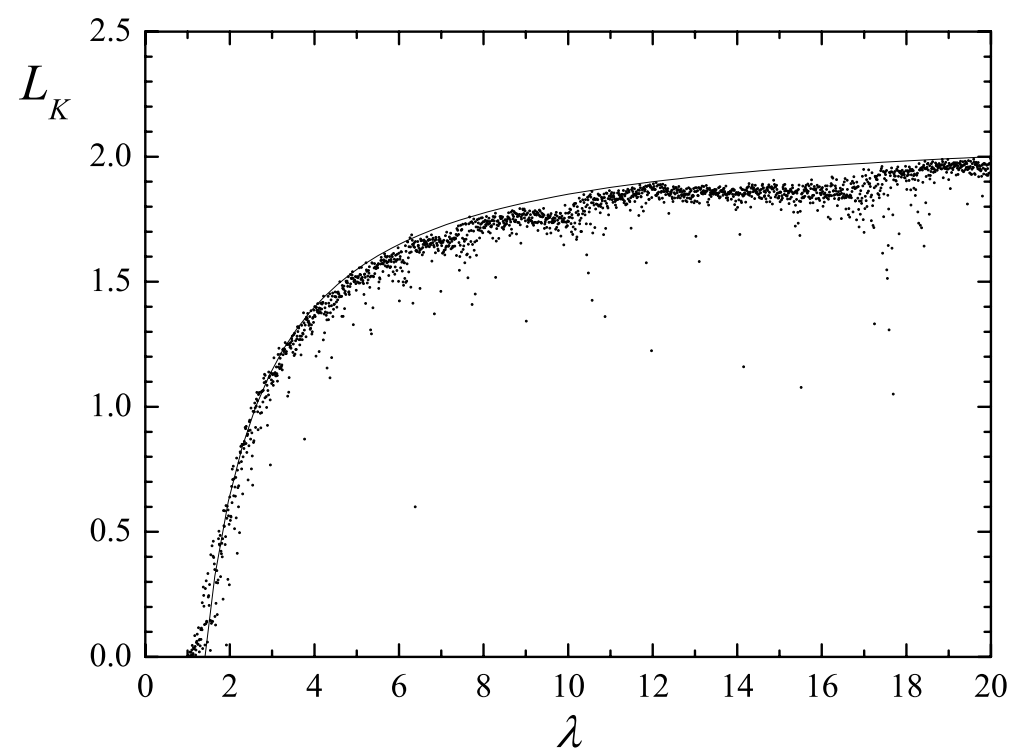

Figure 3. The $\lambda$ dependence of the maximum Lyapunov exponent of the Kepler map and the curve (5.4).

Performing numerical calculation of integral (5.2), we obtain the value of $C_{K} \equiv C_{h}^{g e n}(\gamma=$ $3 / 2)$, that turns out to be equal to $2.21 \ldots$

Let us check the obtained value $C_{K} \approx 2.21$ versus a direct computation of the maximum Lyapunov exponent of the Kepler map. In Fig. 3, the computed maximum Lyapunov exponent of map (5.1) is plotted versus $\lambda$. The limit $\lambda \rightarrow \infty$ of the dependence gives Chirikov's constant; one can see that the computed dependence is in accord with this prediction. All the observed data are below the line $C_{K}=2.2$, as expected. A good fit to the computed dependence is given by the function

$$
L_{K}(\lambda)=C_{K}-\frac{3}{\lambda}
$$

where, however, $C_{K}=2.15$. Therefore, for usage in applications, it is prudent to set $C_{K} \approx 2.2$.

The derivation of the Lyapunov time estimate for a highly-eccentric object is based on a consideration of the Kepler map as a separatrix map, the unperturbed parabolic trajectory playing the role of the separatrix. Then, the Lyapunov time estimate is given by the relation

$$
T_{L} \approx \frac{T_{o r b}}{L_{K}(\lambda)}
$$

where $T_{\text {orb }}$ is the average orbital period of the object. The lower bound for the Lyapunov time is just

$$
T_{L} \approx \frac{T_{o r b}}{C_{K}}
$$

This value of $T_{L}$ corresponds to the motion with $\lambda \gg 1$.

The cradle cause of dynamical chaos in this setting of the problem lies not in "close encounters", as is often physically interpreted, but in the overlapping of resonances in phase space of the Kepler map. So, the intrinsic source of chaos - the resonant interaction for the orbital motion of planet-encountering bodies and for the motion of ordinary asteroids is one and the same. 


\subsection{Lower bounds for the Lyapunov times of NEAs and comets}

The lower bound for the Lyapunov time of the orbital motion of a planet-encountering body is given by relation (5.6). For the comet $1 \mathrm{P} /$ Halley, with the orbital period of 76 years, the lower bound, given by this formula, is about 34 years. Using the data (Chirikov \& Vecheslavov (1986), Vecheslavov \& Chirikov (1988), Chirikov \& Vecheslavov (1989)) on the amplitude of perturbation of the full energy of the comet, one finds $\lambda \approx 1.2 \cdot 10^{4} \gg 1$; consequently, the found lower bound for $T_{L}$ is close to the expected $T_{L}$ value itself.

It is very probable that relation (5.6) applies for the motion of any long-periodic comet, or any Halley-type comet, or any asteroid in a highly-eccentric orbit with a similar kind of perturbation; i.e., the value of the Lyapunov time of such an object is determined solely by its orbital period.

Whipple (1995) explored the chaotic orbital motion of 175 real asteroids with $q<$ 1.6 AU (in the inner part of the Solar system). He found that the Lyapunov times can be as small as 10 years, due to encounters with the terrestrial planets. The range of values of $T_{L}$ is $10-20000$ years. 34 of these 175 objects are so chaotic, that the errors in determination of their orbits double in less than 70 years.

To our present knowledge, no known asteroid or comet violates the bound (5.6). In this respect, the diagram "Lyapunov time - semimajor axis", constructed by Whipple (1995) (see Fig. 2 in his paper), is of particular interest: one can see that all the considered objects have $T_{L} \geqslant 10$ years, and, since they all have the semimajor axis $a<3.5 \mathrm{AU}$, bound (5.6) is in no way violated.

Tancredi (1995), Tancredi (1999) considered the orbital evolution of 145 Jupiter family comets and 307 NEAs (inactive objects with aphelia $Q>1$ AU and perihelia $q<$ $1.5 \mathrm{AU})$. He found $T_{L}$ values in the range 30-200 years for the first group of objects, and 10-300 years, mostly 50-150 years, for the second one. The minimum observed value was $\approx 10$ years.

Thus the planet-encountering asteroids and comets are among the most chaotic objects of the Solar system; their Lyapunov times can be as low as several years. This is in accord with the simple estimate (5.6).

\section{Conclusions}

In this report, we have addressed the problem of predictability of the chaotic asteroidal motion. Up to the present moment, large numerical material on the Lyapunov times of the chaotic asteroidal motion has been accumulated in literature. In overwhelming majority, these estimates were obtained by means of numerical integration. In view of necessity of theoretical explanation of these data, we have presented a method of analytical estimation of the maximum Lyapunov exponents of the orbital motion of asteroids. It is based on the separatrix map theory.

We have considered the chaotic asteroidal motion close to the ordinary and three-body mean motion resonances with planets, and the motion in highly eccentric orbits subject to moderately close encounters with planets. For the case of the mean motion resonances, we have derived simple analytical formulas for the Lyapunov time in four basic resonance type models: the fastly chaotic resonance triad, fastly chaotic resonance duad, slowly chaotic resonance triad, slowly chaotic resonance duad. For the case of highly-eccentric objects subject to moderate encounters with planets, we have derived simple analytical formulas for the Lyapunov time and its lower bound.

The analytical estimates of the Lyapunov times for model and real asteroids have been made and compared to many known numerical ones, i.e., to known estimates obtained 
by means of numerical integration of orbits, including estimates obtained for real objects in the full problem of motion in the Solar system. In many cases a satisfactory agreement have been observed, that testifies the quality of the corresponding theoretical models of asteroidal motion. On the other hand, the cases of disagreement are even more interesting: they may imply either an imperfectness of the adopted perturbed pendulum model as applied to the considered resonance (as noted in Section 4.1 in relation to the $3 / 1$ mean motion resonance), or an incorrect identification of the guiding resonance. So, one can conclude that the analytical estimation of the Lyapunov times may represent a promising tool for discerning between possible models of chaos in the motion of real asteroids, and, generally, celestial bodies.

\section{Acknowledgements}

It is a pleasure to acknowledge the useful comments of a referee. This work was partially supported by the Russian Foundation for Basic Research (project \# 05-02-17555) and by the Programme of Fundamental Research of the Russian Academy of Sciences "Fundamental Problems in Nonlinear Dynamics". The computations were partially carried out at the St. Petersburg Branch of the Joint Supercomputer Center of the Russian Academy of Sciences.

\section{References}

Bretagnon, P. 1990, Astron. Astrophys. 231, 561

Chirikov, B. V. 1977, Nonlinear Resonance (Novosibirsk: Izdatel'stvo NGU) (In Russian)

Chirikov, B. V. 1979, Phys. Rep. 52, 263

Chirikov, B. V. \& Shepelyansky, D. L. 1984, Physica D 13, 395

Chirikov, B. V. \& Vecheslavov, V. V. 1986, INP Preprint 86-184

Chirikov, B. V. \& Vecheslavov, V. V. 1989, Astron. Astrophys. 221, 146

Froeschlé, Cl. 1984, Celest. Mech. 34, 95

Hairer, E., Nørsett, S. P. \& Wanner, G. 1987, Solving Ordinary Differential Equations I. Nonstiff Problems (Berlin: Springer-Verlag)

Holman, M. J. \& Murray, N. W. 1996, Astron. J. 112, 1278

Knežević, Z. \& Milani, A. 2000, Preprint

Kouprianov, V. V. \& Shevchenko, I. I. 2003, Astron. Astrophys. 410, 749

Lichtenberg, A. J. \& Lieberman, M. A. 1992, Regular and Chaotic Dynamics (New York: Springer-Verlag)

Milani, A. \& Nobili, A. M. 1993, Celest. Mech. Dyn. Astron. 56, 323

Milani, A., Nobili, A. M. \& Knežević, Z. 1997, Icarus 125, 13

Morbidelli, A. \& Nesvorný, D. 1999, Icarus 139, 295

Murray, N. W. \& Holman, M. J. 1997, Astron. J. 114, 1246

Murray, N., Holman, M. \& Potter, M. 1998, Astron. J. 116, 2583

Nesvorný, D. \& Morbidelli, A. 1998, Astron. J. 116, 3029

Nesvorný, D. \& Morbidelli, A. 1999, Celest. Mech. Dyn. Astron. 71, 243

Petrosky, T. Y. 1986, Phys. Letters A 117, 328

Shevchenko, I. I. 1998a, Phys. Letters A 241, 53

Shevchenko, I. I. 1998b, Physica Scripta 57, 185

Shevchenko, I. I. 1999, Celest. Mech. Dyn. Astron. 73, 259

Shevchenko, I. I. 2000a, Izvestia GAO 214, 153 (In Russian)

Shevchenko, I. I. 2000b, J. Exp. Theor. Phys. 91, 615 [ZhETP 118, 707]

Shevchenko, I. I. 2002, Cosmic Res. 40, 296 [Kosmich. Issled. 40, 317]

Shevchenko, I. I. \& Kouprianov, V. V. 2002, Astron. Astrophys. 394, 663

Shevchenko, I. I., Kouprianov, V. V. \& Melnikov, A. V. 2003, Solar System Res. 37, 74 [Astronomicheskii Vestnik 37, 80] 
Shevchenko, I. I. 2004a, in: G. Byrd et al. (eds.), Order and Chaos in Stellar and Planetary Systems, ASP Conf. Series, vol. 316, p. 20

Shevchenko, I. I. 2004b, JETP Letters 79, 523 [Pis'ma Zh. Eksp. Teor. Fiz. 79, 651]

Tancredi, G. 1995, Astron. Astrophys. 299, 288

Tancredi, G. 1999, Celest. Mech. Dyn. Astron. 70, 181

Vecheslavov, V. V. \& Chirikov, B. V. 1988, Sov. Astron. Letters 14, 151

von Bremen, H. F., Udwadia, F. E. \& Proskurowski, W. 1997, Physica D 101, 1

Whipple, A. L. 1995, Icarus 115, 347

Wisdom, J. 1983, Icarus 56, 51 